Editorial

wissen kompakt 2019 $13: 171$

https://doi.org/10.1007/s11838-019-00097-7

(c) Freier Verband Deutscher Zahnärzte (FVDZ) and Springer Medizin Verlag $\mathrm{GmbH}$, ein Teil von Springer Nature 2019

\author{
P. W. Kämmerer' ·. M. Stein ${ }^{2}$ \\ ${ }^{1}$ Klinik und Poliklinik für Mund-, Kiefer- und Gesichtschirurgie, plastische Operationen, \\ Universitätsmedizin, Johannes Gutenberg-Universität Mainz, Mainz, Deutschland \\ ${ }^{2}$ Klinik für Zahnerhaltung, Parodontologie und Präventive Zahnheilkunde, Universitätsklinikum, RWTH \\ Aachen, Aachen, Deutschland
}

\title{
Mit Blutungen richtig umgehen
}

In der täglichen zahnärztlichen Behandlung kommt es immer wieder zu kleineren Blutungsereignissen, die mitunter den Zahnarzt und den Patienten verunsichern und verängstigen können. Nach Verletzungen, bei denen es zur Eröffnung kleinerer Blutgefäße gekommen ist, sistiert die Blutung beim Gesunden i. Allg. nach wenigen Minuten von selbst. Unter einer Nachblutung versteht man die nach fachgerechter Versorgung einer Wunde auftretende Blutung, die sich klinisch in einer diffusen Blutung aus Schleimhauträndern, submukösem Gewebe und Knochen ohne erkennbare Gefäßpulsation manifestieren kann. Die Nachblutung kann entweder als Früh- oder auch als Spätblutung - mit einer zeitlichen Verzögerung von Tagen - in Erscheinung treten, wobei beim Vorkommen von Blutungen nicht zwangsläufig auf eine Manifestation von Gerinnungsstörungen geschlussfolgert werden kann. So wird beispielsweise auch bei gesunden Patienten durch den vasokonstriktorischen Zusatz des Lokalanästhetikums eine reaktive Hyperämie mit konsekutiv einsetzender Blutung beobachtet. Die Infektion und der Zerfall des Blutkoagulums, eine gelockerte oder gelöste Wundnaht, aber auch eine gesteigerte Fibrinolyse im Rahmen überschießender Plasminaktivität sind ebenso an der Entwicklung einer Nachblutung beteiligt wie postoperatives Fehlverhalten des Patienten. Hier sind v. a. wiederholte Mundspülungen, ein (nervöses) Zungenspiel oder andere mechanische Irritationen an der Wunde, schwere körperliche Arbeit, sportliche Betätigung und auch ein gesteigerter Alkohol- und/oder Kaffeekonsum zu nennen. Im Großteil der Fälle stellen sich diese Blutungskomplikationen als lediglich marginal dar. Beim Erwachsenen kann beispielsweise erst der Verlust von ungefähr einem Liter Blut zu einer lebensbedrohlichen Situation führen; bei Kindern und Kleinkindern besteht diese Gefahr wegen der insgesamt geringeren Blutmenge allerdings schon erheblich früher.

Obwohl Patienten ohne pathologische Veränderungen des Blutgerinnungssystems in der Praxis den größten Anteil an Nachblutungen stellen, sind es gerade Patienten mit kongenitalen oder erworbenen Gerinnungsstörungen, die einem besonderen Blutungsrisiko unterliegen und in der vorliegenden Ausgabe von wissen kompakt besonders thematisiert werden. Die deutlich verbesserte Therapierbarkeit kardiovaskulärer und thromboembolischer Erkrankungen hat zu einer höheren Lebenserwartung beigetragen; damit steigt allerdings auch die Zahl und das Alter der Patienten, die sich unter einer antikoagulativen/ thrombozytenggregatorischen Therapie zahnärztlichen Eingriffen unterziehen. Der niedergelassene Zahnarzt muss durch sorgfältige Erhebung der Anamnese Risikopatienten sicher erfassen. Gliedert man die hämorrhagischen Diathesen nach ihren Ursachen, werden gleichzeitig wesentliche Aspekte für die Risikoabschätzung und Erfordernisse für die individuelle Therapie erkannt. Der Umgang mit Blutungen bedingt vom Zahnarzt somit Kenntnis der potenziellen Störungen der Hämostase sowie der entsprechenden lokalen, aber auch systemischen Behandlung. Es obliegt der zahnärztlichen Kompetenz, selektierte Eingriffe vor Ort ambulant durchzuführen oder zur Vermeidung von Komplikationen frühzeitig an eine Fachklinik zu überweisen.

PD Dr. Dr. Peer W. Kämmerer

Prof. Dr. Jamal M. Stein

\section{Korrespondenzadresse}

PD Dr. Dr. P. W. Kämmerer,
MA, FEBOMFS
Klinik und Poliklinik
für Mund-, Kiefer-
und Gesichtschirurgie,
plastische Operationen,
Universitätsmedizin,
Johannes Gutenberg-
Universität Mainz
Augustusplatz 2,
55131 Mainz, Deutschland
peer.kaemmerer@
unimedizin-mainz.de

Interessenkonflikt. P.W. Kämmerer und J. M. Stein geben an, dass kein Interessenkonflikt besteht. 